Pacific Journal of Mathematics

LEFT EUCLIDEAN RINGS 


\title{
LEFT EUCLIDEAN RINGS
}

\author{
H. H. BRungs
}

It is easy to expand the definition of a commutative Euclidean domain to non commutative rings with zero divisors. Using such a generalized definition it is proved that matrix rings over Euclidean domains are Euclidean, that left principal ideal domains with finitely many maximal left ideals only, which are assumed to be two sided, are Euclidean and that direct sums of Euclidean rings are Euclidean. It follows from this that semi simple rings with d.c.c. are Euclidean.

P. Samuel in [10] calls a ring $R$ Euclidean if there exists a map $\phi$ from $R$ into a well ordered set $W$ such that the following property is satisfied:

E. For $a, b$ in $R, b \neq 0$ there exist $q$ and $r$ in $R$ such that $a=q b+r$ with $\phi(r)<\phi(b)$.

This definition applies of course to non commutative rings as well, not only to commutative rings as in [10]. If we want to be precise we will say $R$ is left Euclidean for $\phi$. It will be assumed throughout that every ring $R$ has a unit element.

Non commutative Euclidean rings have been considered much earlier. We have the well known examples by Ore [9] and one knows that under additional conditions on the function $\phi$ Ore's examples are essentially the only ones; see [3].

P. M. Cohn in [3] generalized the Euclidean algorithm to apply it to free algebras and transfinite generalizations of the usual Euclidean algorithm satisfying certain additional conditions where considered by Jategaonkar in [6]; see [5] for a transfinite generalization of the weak algorithm.

We will admit rings with zero divisors in this discussion and show that direct sums of left Euclidean rings and $n \times n$ matrix rings over left Euclidean domains are left Euclidean. From this it follows that semi simple rings are both left and right Euclidean. We prove further that a left principal ideal domain with only finitely many maximal ideals which are assumed to be all two sided is left Euclidean.

One concludes as in the commutative case that a left Euclidean ring is a left principal ideal ring. Further it is obvious by considering examples that a left Euclidean ring is not necessarily right noetherian and therefore not necessarily right Euclidean.

We list some results which carry over immediately from [10]. If $R$ is left Euclidean, $b \neq 0$ in $R$ then $\phi(b)>\phi(0)$. Let $R$ be any 
ring. We define:

$R_{0}=\{0\}$ and $R_{\alpha}$ by transfinite induction as follows:

$$
R_{\alpha}^{\prime}=\bigcup_{\beta<a} R_{\beta} \quad \text { and } R_{\alpha} \text { is the union of }\{0\}
$$

and the set of all elements $b$ in $R$ such that $R_{\alpha}^{\prime} \rightarrow R / R b$ is an onto mapping.

It is clear that if $R$ is left Euclidean there will be an ordinal $\alpha$ such that $R_{\alpha}=R$.

If on the other hand $R$ is reached by some $R_{\alpha}$ in the above construction one can define a left algorithm $\psi$ by setting

$$
\psi(r)=\beta \quad \text { for } r \in R_{\beta} / R_{\beta^{\prime}} .
$$

This smallest algorithm will satisfy the following properties:

(1) $\psi(x)=0 \rightleftarrows x=0$

(2) $\psi(x)=1 \rightleftarrows x$ has a left inverse in $R$.

(3) $\psi(a b) \geqq \psi(b)$ for $a b \neq 0$

(4) $\psi(a)=\inf \psi(b)$ for $b \neq 0$ in $R a \neq 0$.

We will apply the above criterium to prove the following:

THEOREM 1. The ring $R_{n \times n}$ of $n \times n$ matrices over a left Euclidean domain $R$ is a left Euclidean ring.

Let $R$ be a left Euclidean domain for $\phi$. We may assume that $\phi$ satisfies the properties $1-4$ listed above. Every left ideal in $R_{n \times n}$ is a left principal ideal and is generated by an element $M$ of the following standard form: Only the first $k$ rows of $M$ are nonzero. Let $r_{i c_{i}}$ be the first nonzero element in the $i$ th row for $i=1, \cdots, k$. Then we have $1 \leqq c_{1}<c_{2}<\cdots<c_{k} \leqq n$, and the columns to the left of the $c_{1}$ st column are zero, a column between the $c_{i}$ th and $c_{i+1}$ th column has the last $n-i$ elements equal to zero and the columns to the right of the $c_{k}$ th column have the last $n-k$ elements equal to zero.

The elements $r_{j c_{i}}$ are zero for $j>i$ and $\phi\left(r_{j c_{i}}\right)<\phi\left(r_{i c_{i}}\right)$ for $j<i$. If for a matrix $N$ in $R_{n \times n}$ the left principal ideal generated by $N$ is generated by a matrix $M$ in standard form as described above then we will say $N \in A_{r_{c_{1}}} \cdots{ }_{r_{c_{k}}}$ with $r_{i_{i}}=r_{c_{i}} ; A_{0}=$ \{zero matrix $\}$.

The integers $k, c_{1}, \cdots, c_{k}$ are uniquely determined by the left ideal $I$ of $R_{n \times n}$ and the elements $r_{i c_{i}}$ are uniquely determined up to units in $R$ as left factors. One sees this when one recalls the proof of the fact that $R_{n \times n}$ is a left principal ideal ring. Every left ideal in $I$ is generated by a matrix $B$ in which the first $i-1$ elements in 
the $i$ th row are zero, and the element in the $i i$-position is a generator of the left ideal in $R$ consisting of the elements appearing in the $i$ th column of matrices of $I$ with zero $j$ th column for $j<i$. If this element is zero the whole row can be chosen to be zero. Interchanging rows leads then to the matrix in standard form as described above.

We now order the sets $A_{r_{c_{i}}} \cdots{ }_{r_{c_{k}}}$, and we say that $A_{a_{t_{1}}} \cdots_{a_{t_{k}}}$ is earlier then $A_{b_{s_{1}}} \cdots{ }_{b_{s_{k}}}$ if either

(i) $h>k$ or

(ii) $h=k$ and $t_{1}=s_{1}, \cdots, t_{i}=s_{i}$, but $t_{i+1}<s_{i+1}$ or

(iii) $h=k, t_{i}=s_{i}$ for $i=1, \cdots, k, \phi\left(a_{t_{1}}\right)=\phi\left(b_{s_{1}}\right), \cdots, \phi\left(a_{t_{i}}\right)=\phi\left(b_{s_{i}}\right)$, but $\phi\left(a_{t_{i+1}}\right)<\phi\left(b_{s_{i+1}}\right)$.

To prove now that $R_{n \times n}$ is left Euclidean it is sufficient that for $a$ in $R_{n \times n}, 0 \neq b \in R_{n \times n}$ an element $q$ in $R_{n \times n}$ exists such that $a-q b$ is in a set $A_{r_{c_{1}}} \cdots r_{c_{k}}$ which is earlier then the corresponding set of $b$. This will guarantee that the transfinite construction described above reaches eventually the ring $R_{n \times n}$.

We denote by $R^{n}$ the free $R$ left module of rank $n$. If $B$ is an element in $R^{n}$ and $1 \leqq m \leqq n$ we denote with $B^{m}$ the element in $R^{m}$ whose components are the first $m$ components of $B$. As usual, we will say that $A_{1}, \cdots, A_{t} \in R^{n}$ are linearly independent if $\sum r_{i} A_{i}=0$ implies $r_{i}=0$ for all $i$ and $r_{i}$ elements in $R$.

We need a Lemma before we can prove the theorem.

Lemma. Assume three sets $\left\{B_{1}, \cdots, B_{s}\right\},\left\{M_{1}, \cdots, M_{k}\right\},\left\{A_{1}, \cdots, A_{k}\right\}$ of elements in $R^{n}$ are given such that $\left\{B_{1}, \cdots, B_{s}, M_{1}, \cdots, M_{k}\right\}$ is a linearly independent set. Then there exist $\varepsilon_{j}=0$ or 1 for $j=$ $1,2, \cdots, k$ such that $\left\{B_{1}, \cdots, B_{s}, B_{s+1}, \cdots, B_{s+k}\right\}$ is linearly independent for $B_{s+j}=A_{j}+\varepsilon_{j} M_{n_{j}}$.

Assume for a proof that $B_{1}, \cdots, B_{s}, B_{s+1}, \cdots, B_{s+j}$ are constructed and linearly independent for some $0 \leqq j<k$. If $\left\{B_{1}, \cdots, B_{s+j}, A_{j+1}\right\}$ is a linearly independent set, let $\varepsilon_{j+1}=0$ and $B_{s+j+1}=A_{j+1}$. Otherwise we conclude that the rank of the left $R$-module $N$ generated by $B_{1}, \cdots, B_{s}, B_{s+1}, \cdots, B_{s+j}, A_{j+1}$ is equal to $s+j$. If we assume for every $i=1, \cdots, k$ that elements $r_{j+1, i}^{\prime} \neq 0$ and $r_{t, i}$ in $R$ exist for $t=1, \cdots, s+j$ such that

$$
r_{j+1, i}^{\prime}\left(A_{j+1}+M_{i}\right)=\sum_{t=1}^{s+j} r_{t, i} B_{t}
$$

then we conclude that the module $N$ contains a free submodule of rank $s+k>s+j=\operatorname{rank} N$ generated by $B_{1}, \cdots, B_{s}, v M_{1}, \cdots, v M_{k}$ 
with $v \neq 0$, a left common multiple of the $r_{j+1, i}^{\prime}$ for $i=1, \cdots, k$. This is impossible (as follows from earlier remarks or see [3]) and proves the Lemma.

We are now ready to prove the theorem.

Let $a$ be any matrix in $R_{n \times n}, \quad b$ a nonzero matrix in $A_{r_{c_{1}}} \cdots_{r_{c_{k}}}$. Then there exists a matrix $q^{\prime}$ in $R_{n \times n}$ such that $a-q^{\prime} b=r^{\prime}$ is a matrix in whose $c_{i}$ th column only elements with $\phi$-value smaller than $\phi\left(r_{c_{i}}\right)$ appear. We may assume that $b$ is already in standard form, and we will show that by adding suitable rows of the matrix $b$ to rows of $r^{\prime}$ the resulting matrix appears in some earlier set $A_{s_{n_{1}}} \cdots_{s_{n_{t}}}$ than $b$.

The following can happen:

(a) $r^{\prime}$ is the zero matrix and we are finished.

(b) The first nonzero column of $r^{\prime}$ is not one of the $c_{i}$-columns, $i=1, \cdots, k$. Then let $B_{1}$ be a row of $r^{\prime}$ with a nonzero element in the first nonzero column of $r^{\prime}$.

Let $M_{1}, \cdots, M_{k}$ be the rows of $b$ and let $A_{1}, \cdots, A_{k}$ be any $k$ rows of $r^{\prime}$ different from $B_{1}$. Then we can apply the lemma and we obtain a matrix

$$
r^{\prime}-q^{\prime \prime} b=r
$$

which has rank at least $k+1$.

(c) The first nonzero column of $r^{\prime}$ is the $c_{i}$ th column for some $1 \leqq i \leqq k$. Choose $B_{1}$ as any row of $r^{\prime}$ with a nonzero element $y$ in the $c_{i}$ th spot. Let $A_{2}, \cdots, A_{i}$ be any $i-1$ rows of $r^{\prime}$ different from $B_{1}$ and let $M_{1}, \cdots, M_{i-1}$ be the first $i-1$ rows of $b$. Set

$$
B_{e+1}=A_{e+1}+M_{e}
$$

for $e=1, \cdots, i-1$ to obtain a linearly independent set

$$
\left\{B_{1}^{c_{i}}, \cdots, B_{i}^{c_{i}}\right\} \text {. }
$$

We distinguish now two subcases:

(i) $c_{i+1}=c_{i}+1, \cdots, c_{i+e}=c_{i}+e$, but $c_{i+e+1}>c_{i}+e+1$. We consider $B_{1}^{w}, \cdots, B_{i}^{w}, M_{i+1}^{w}, \cdots, M_{i+e}^{w}$, i.e. elements in $R^{w}$ for $w=$ $c_{i}+e$, where $M_{j}$ is the $j$ th row of $b$.

Choose any $e$ rows of $r^{\prime}$ different from $B_{1}, A_{2}, \cdots, A_{i}$ and obtain by the lemma $B_{1}, \cdots, B_{i}, B_{i+1}, \cdots, B_{i+e}$ such that $\left\{B_{1}^{w}, \cdots, B_{i+e}^{w}\right\}$ is a linearly independent set. 
(ii) $c_{i}+1<c_{i+1}, c_{i+2}=c_{i+1}+1, \cdots, c_{i+h}=c_{i+1}+h-1$, but $c_{i+h+1}>c_{i+1}+h$.

In this case consider $B_{1}^{w}, \cdots, B_{i}^{w}, M_{i+1}^{w}, \cdots, M_{i+h}^{w}$ as in (i) but for $w=c_{i+h}$. Choose any $h$ rows of $r^{\prime}$ different from $B_{1}, A_{2}, \cdots, A_{i}$ and obtain by the lemma $B_{1}, \cdots, B_{i}, B_{i+1}, \cdots, B_{i+h}$ such that

$$
\left\{B_{1}^{w}, \cdots, B_{i}^{w}, B_{i+1}^{w}, \cdots, B_{i+h}^{w}\right\}
$$

is a linearly independent set.

Repeating step $c$, (ii) we can build a matrix $r$ with

$$
r=r^{\prime}-q^{\prime \prime} b
$$

which is contained in an earlier set $A_{s_{n_{1}}} \cdots_{s_{n_{t}}}$ than $b$. This proves the theorem.

COROLLARY. If $R$ is a division ring then $R_{n \times n}$ is left (and right) Euclidean with $\phi(M)=n-\operatorname{rank}(M)+1$ if $M \neq 0$ and $\phi(0)=0$.

The proof of the next result carries over from [10], Prop. 6 to the non commutative case:

THEOREM 2. The direct sum of finitely many left Euclidean rings is left Euclidean.

We obtain with the corollary to Theorem 1:

COROllary. A semi simple ring $R$ is left (and right) Euclidean.

Finally, we prove a non commutative version of Prop. 5 in [10].

THEOREM 3. A left principal ideal domain $R$ with only finitely many maximal left ideals which are assumed to be two sided is left Euclidean.

In order to prove this theorem we have to recall certain results from Chapter III in [8].

Let $R$ be a ring satisfying the conditions of Theorem 3 . The prime ideals of $R$ contained in one of the maximal ideals $M_{i}$ are the transfinite powers $M_{i}^{\omega^{\alpha}}$. If for a prime ideal $0 \neq P=M_{i}^{\omega^{\alpha}}$, then $\alpha$ is uniquenly determined. We say $P$ has $M_{i}$ weight $\alpha$ and we say $P$ has weight $\alpha$ if $\alpha$ is maximal among the $M_{i}$-weights.

Using the fact that two prime ideals $P_{1}$ and $P_{2}$ with $P_{1} \nsubseteq P_{2} \nsubseteq P_{1}$ 
commute and that $P_{2}^{\prime} P_{1}^{\prime}=P_{2}^{\prime}$ for prime ideals $P_{2}^{\prime} \subsetneq P_{1}^{\prime}$ one can write every non unit $a \neq 0$ in $R$ as a product of prime elements, i.e. elements that generate prime ideals $\neq(0), \neq R$, in a certain standard way; or in Jategaonkar's language:

$$
a=p_{1} \cdots p_{n} \text { such that }\left(p_{1}, \cdots, p_{n}\right)
$$

is an admissable set of prime elements.

Such a factorization is unique up to permissible rearrangements and associates. $R$ is left Euclidean for $\phi$ defined by

$$
\phi(a)=\omega^{\alpha_{1}} m_{1}+\cdots+\omega^{\alpha_{k}} m_{k}
$$

where $m_{i}$ is the number of primes of weight $\alpha_{i}$ in a standard factorization of $a ; \phi$ (unit) $=0, \phi(0)=-1$.

To prove this consider any two elements $a, b \neq 0$ in $R$. If

$$
R a+R b=R,
$$

no common prime appears in any standard factorization of $a$ and $b$.

Assume $\left\{M_{i}^{\prime}\right\}=\left\{M_{i} ; a \in M_{i}\right\} ;\left\{M_{j}^{\prime \prime}\right\}=\left\{M_{j} ; b \in M_{j}\right\}$ and let $p_{1}, \cdots, p_{k}$ be generators of the maximal prime ideals different from all $M_{i}^{\prime}$ and all $M_{j}^{\prime \prime}$. We conclude that $a-p_{1} \cdots p_{k} b$ is a unit and we are done. (We excluded the trivial cases $a=0$ or $b$ a unit.)

If now $R a+R b=R d=R b$ we are finished again and only the case $R a+R b=R d \supsetneqq R b$ remains.

Then $a=a_{1} d, b=b_{1} d$ and there exists $q$ in $R$ and a unit $u$ such that

$$
a_{1}=q b_{1}+u \text {; it follows that } a=q b+u d \text { and } \phi(u d)<\phi(b) .
$$

This last inequality is true since $\phi(p d)>\phi(\alpha)$ for any prime element $p$ in $R$.

A special class of rings which satisfy the conditions of Theorem 3 are the non commutative Krull domains with finitely many defining overrings only. For examples see [2], and for examples of local rings in which primes of arbitrary weight appear see [6].

It follows further from the results in [1] that rings in which all left ideals are inversely well ordered by inclusion are left Euclidean rings. This expands at least for the local case the result in Theorem 
3 to certain rings with zero divisors.

\section{REFERENCES}

1. H. H. Brungs, Generalized discrete valuation rings, Canad. J. Math., 21 (1969), 1404-1408.

2. - Non commutative Krull domains, J. Reine Angew Math.

3. P. M. Cohn, On a generalization of the Euclidean algorithm, Proc. Cambridge Phil. Soc., 57 (1961), 18-30.

4. - Free ideal rings, J. Algebra, 1 (1964), 47-69.

5. - Rings with a transfinite weak algorithm, Bull. London Math. Soc., 1 (1969), 55-59.

6. A. V. Jategaonkar, $A$ counter example in ring theory and homological algebra, J. Algebra, 12 (1969), 418-440.

7. — Rings with transfinite left division algorithm, Bull. Amer. Math. Soc., 75 (1969), 559-561.

8. - Left Principal Ideal Rings, Lecture notes in mathematics No. 123, Springer Verlag, Berlin, Heidelberg, New York 1970.

9. O. Ore, Theory of non commutative polynomials, Ann. of Math., 34 (1933), 480-508. 10. P. Samuel, About Euclidean rings, J. Algebra, 19 (1971), 282-301.

Received December 16, 1971.

UNIVERSITY OF ALBERTA 



\section{PACIFIC JOURNAL OF MATHEMATICS}

EDITORS

\author{
H. SAMELSON \\ Stanford University \\ Stanford, California 94305 \\ C. R. HOBBY \\ University of Washington \\ Seattle, Washington 98105
}

\author{
J. DUGUNDJI \\ Department of Mathematics \\ University of Southern California \\ Los Angeles, California 90007 \\ RICHARD ARENS \\ University of California \\ Los Angeles, California 90024
}

\section{ASSOCIATE EDITORS}

E. F. BECKENBACH

B. H. NeUManN

F. WOLF

K. YosHIDA

\section{SUPPORTING INSTITUTIONS}

UNIVERSITY OF BRITISH COLUMBIA

CALIFORNIA INSTITUTE OF TECHNOLOGY

UNIVERSITY OF CALIFORNIA

MONTANA STATE UNIVERSITY

UNIVERSITY OF NEVADA

NEW MEXICO STATE UNIVERSITY

OREGON STATE UNIVERSITY

UNIVERSITY OF OREGON

OSAKA UNIVERSITY
UNIVERSITY OF SOUTHERN CALIFORNIA

STANFORD UNIVERSITY

UNIVERSITY OF TOKYO

UNIVERSITY OF UTAH

WASHINGTON STATE UNIVERSITY UNIVERSITY OF WASHINGTON

AMERICAN MATHEMATICAL SOCIETY NAVAL WEAPONS CENTER 


\section{Pacific Journal of Mathematics}

\section{Vol. 45, No. $1 \quad$ September, 1973}

William George Bade, Complementation problems for the Baire classes .......... 1

Ian Douglas Brown, Representation of finitely generated nilpotent groups ........ 13

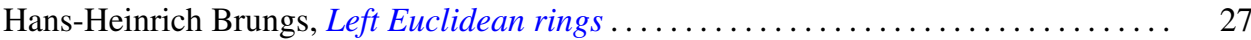

Victor P. Camillo and John Cozzens, A theorem on Noetherian hereditary rings ..... 35

James Cecil Cantrell, Codimension one embeddings of manifolds with locally flat

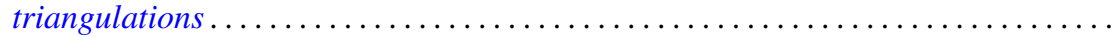

L. Carlitz, Enumeration of up-down permutations by number of rises . . . . . . . . . .

Thomas Ashland Chapman, Surgery and handle straightening in Hilbert cube

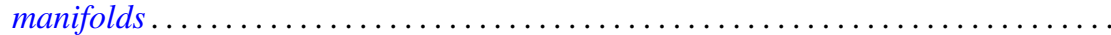

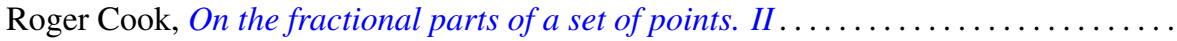

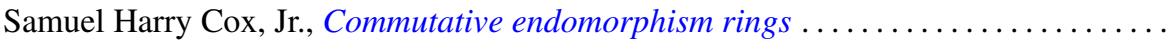

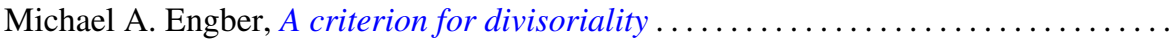

Carl Clifton Faith, When are proper cyclics injective . . . . . . . . . . . . . . 97

David Finkel, Local control and factorization of the focal subgroup . . . . . . . . . 113

Theodore William Gamelin and John Brady Garnett, Bounded approximation by

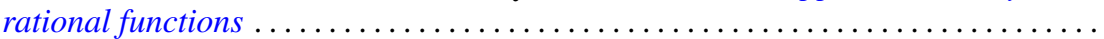

Kazimierz Goebel, On the minimal displacement of points under Lipschitzian

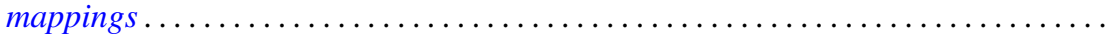

Frederick Paul Greenleaf and Martin Allen Moskowitz, Cyclic vectors for representations associated with positive definite measures: nonseparable

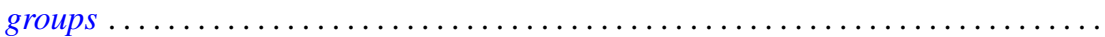

Thomas Guy Hallam and Nelson Onuchic, Asymptotic relations between perturbed linear systems of ordinary differential equations .

David Kent Harrison and Hoyt D. Warner, Infinite primes of fields and completions. .

James Michael Hornell, Divisorial complete intersections . ......

Jan W. Jaworowski, Equivariant extensions of maps ..............

John Jobe, Dendrites, dimension, and the inverse arc function .. .

Gerald William Johnson and David Lee Skoug, Feynman integrals of non-factorable

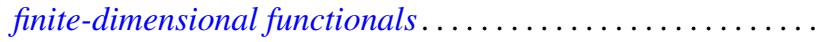

Dong S. Kim, A boundary for the algebras of bounded holomorphic functions ...... 269

Abel Klein, Renormalized products of the generalized free field and its derivatives ... 275

Joseph Michael Lambert, Simultaneous approximation and interpolation in $L_{1}$ and

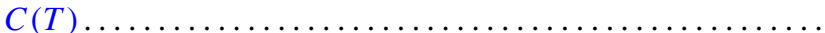

Kelly Denis McKennon, Multipliers of type $(p, p)$ and multipliers of the group

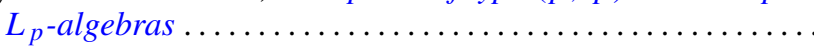

William Charles Nemitz and Thomas Paul Whaley, Varieties of implicative

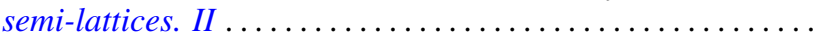

Donald Steven Passman, Some isolated subsets of infinite solvable

Norma Mary Piacun and Li Pi Su, Wallman compactifications on E-completely

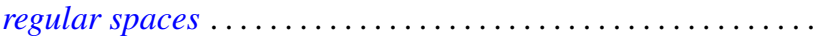

Jack Ray Porter and Charles I. Votaw, $S(\alpha)$ spaces and regular Hausdorff extensions....

Gary Sampson, Two-sided $L_{p}$ estimates of convolution transforms .

Ralph Edwin Showalter, Equations with operators forming a rig
Raymond Earl Smithson, Fixed points in partially ordered sets .

Victor Snaith and John James Ucci, Three remarks on symmetric products and

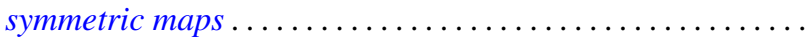

